Supporting Information

\title{
Bowl-Like Nanoreactor Composed of Packed Gold Nanoparticles Surrounded with Silica Nanosheets for Photothermally Enhancing Enzymatic Reaction
}

Jiao Sun, ${ }^{\dagger}$ Chen Cao, ${ }^{\S}$ Jun Chen, ${ }^{\dagger}$ Wenli Zhao, ${ }^{\dagger}$ Qin Tang,,$^{\dagger}$ Bohong Jiang, ${ }^{\dagger}$ Fan Xu, ${ }^{\dagger}$ Yehao Zhang, ${ }^{\dagger}$ and Qingmin $\mathrm{Ji}^{\dagger *}$

$\dagger$ Herbert Gleiter Institute for Nanoscience, School of Materials Science and Engineering, Nanjing University of Science \& Technology, 200 Xiaolingwei, Nanjing, 210094, China.

$\S$ School of Chemical Engineering, Nanjing University of Science \& Technology, 200 Xiaolingwei, Nanjing, 210094, China.

$\ddagger$ Institute of Optoelectronics \& Nanomaterials, MIIT Key Laboratory of Advanced Display Materials and Devices, Nanjing University of Science and Technology, 200 Xiaolingwei, Nanjing, 210094, China.

Corresponding author: jiqingmin@ njust.edu.cn 


\section{Additional Data}

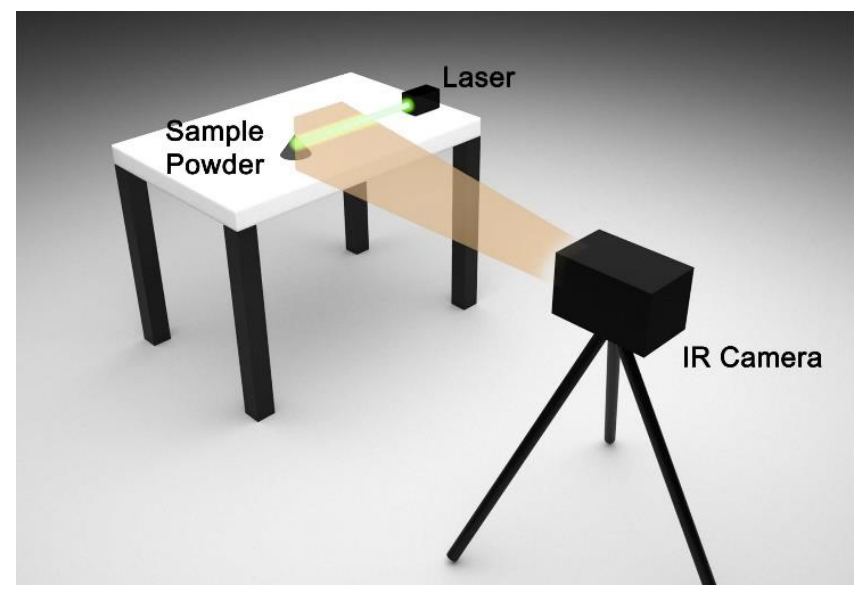

Figure S1. The scheme for the photothermal experimental setup composed of a laser beam irradiating on the sample powder surface and an IR camera to record the spatial temperature in real time.

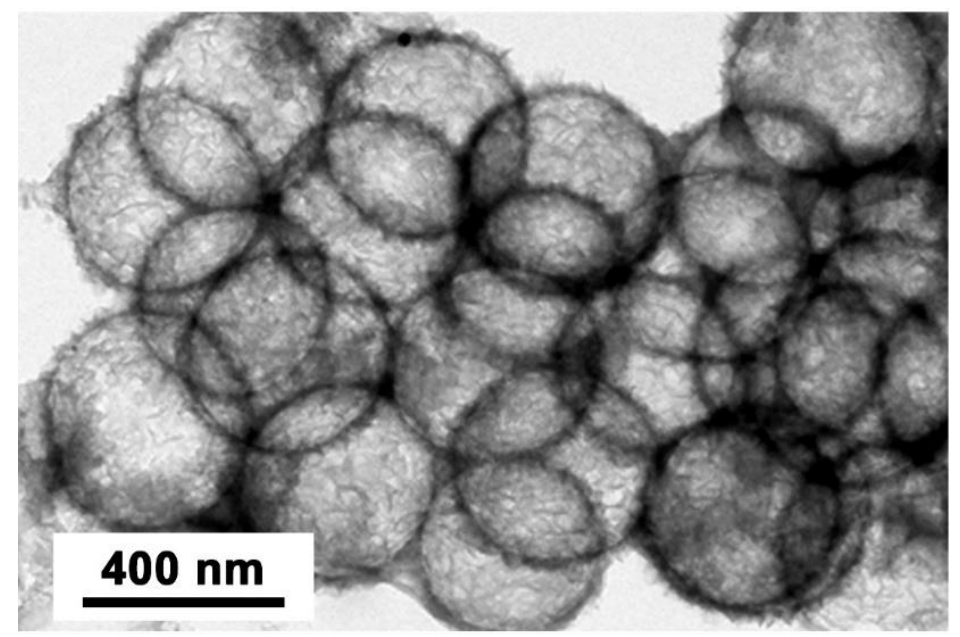

Figure S2. The TEM image of hollow silica spheres from the hydrothermal reaction of silica particles in $\mathrm{NaBH}_{4}$ solution. 

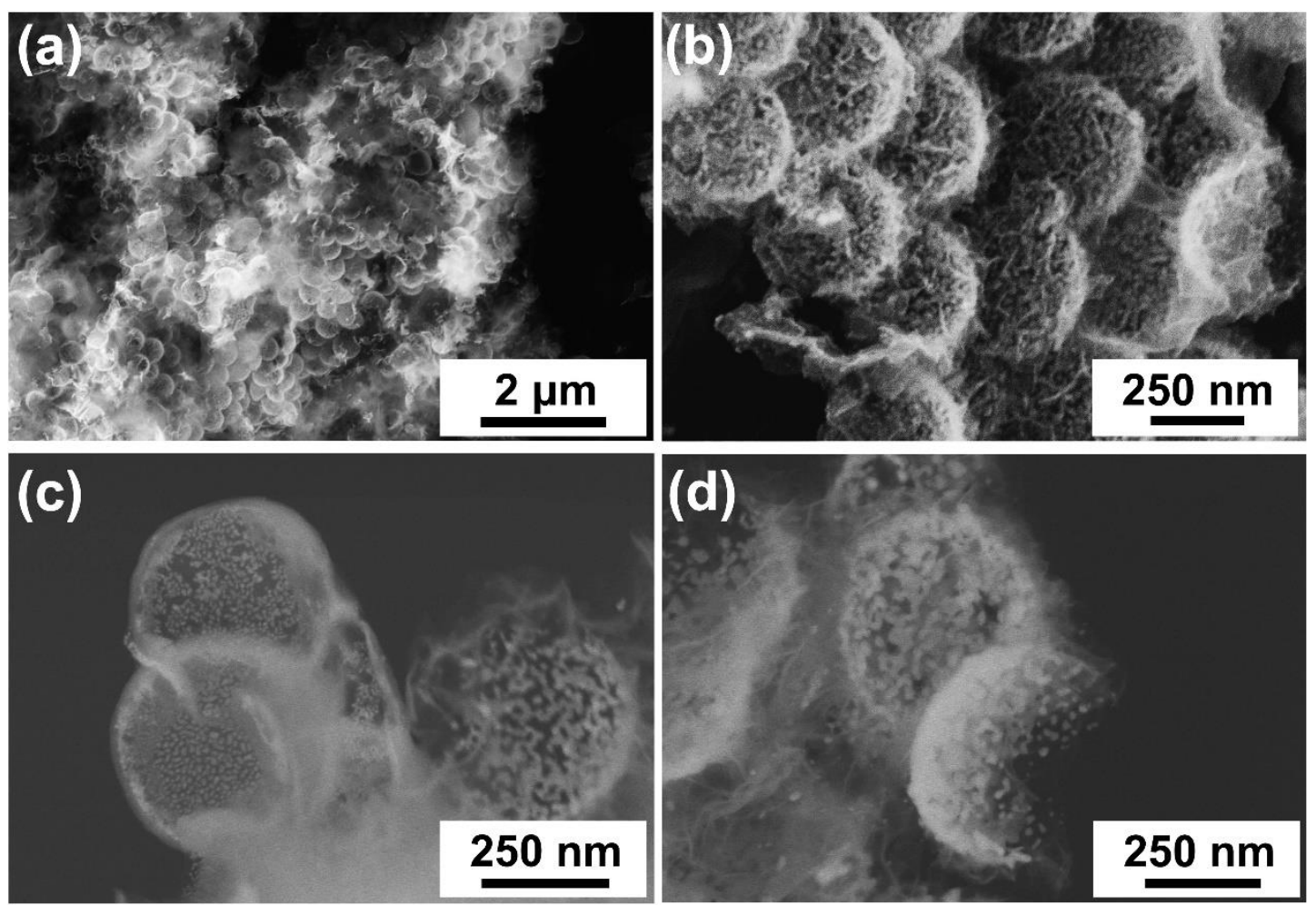

Figure S3. (a-b) The SEM images, (c) STEM image and (d) TEM image of the bowl-

like spheres with separated packed Au NPs and silica nanosheets. 


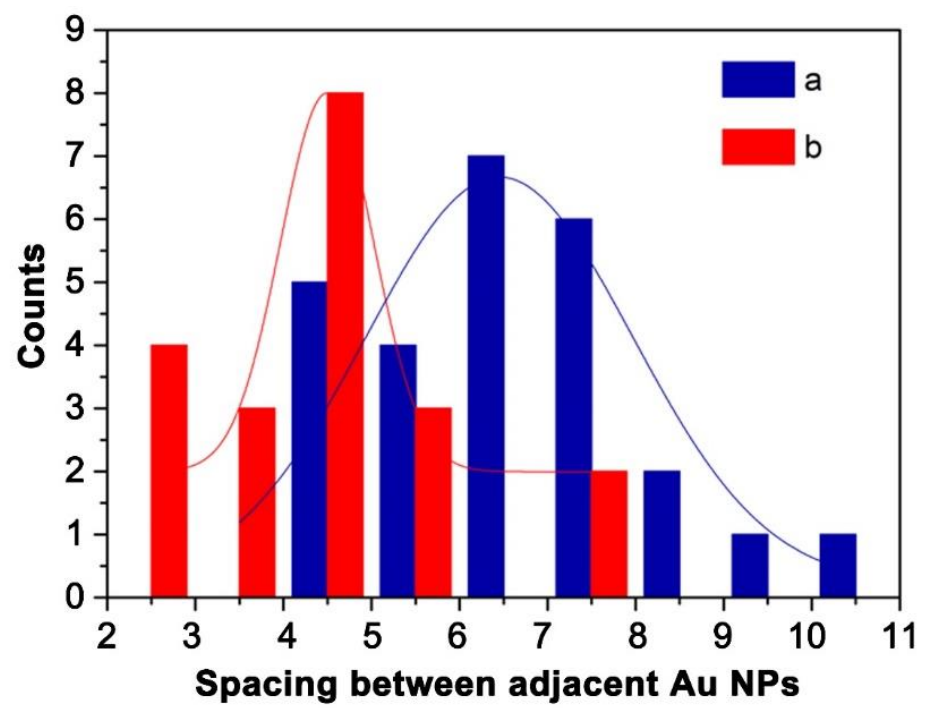

Figure S4. The comparison of the distances between Au NPs before (on silica sphere) and after (in bowl-like spheres) hydrothermal synthesis. The measured spacings (based on the TEM observations) between adjacent Au NPs (a) in the bowl-like spheres and (b) in the sputtered Au layer on the silica particles. 


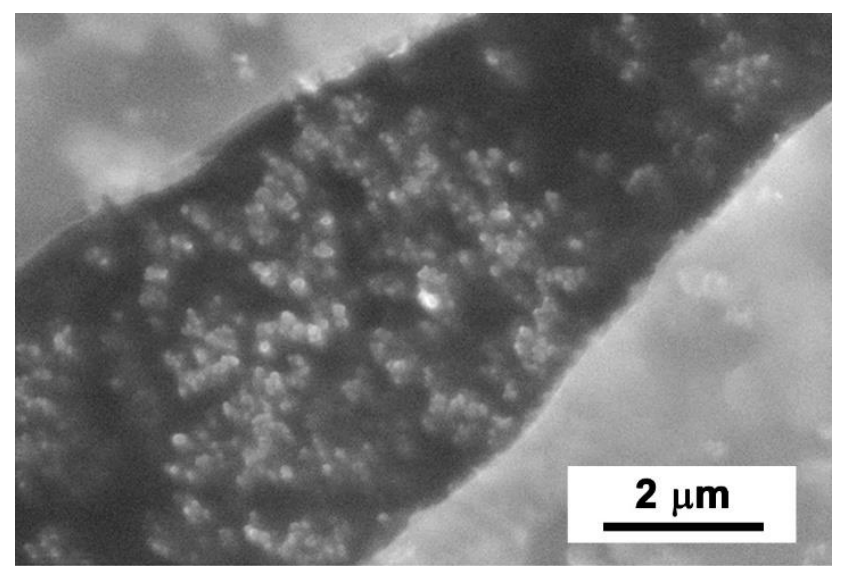

Figure S5. The SEM image of the silica particles with sputtered Au layer after immersing into the diluted HF solution for 2 minutes. 


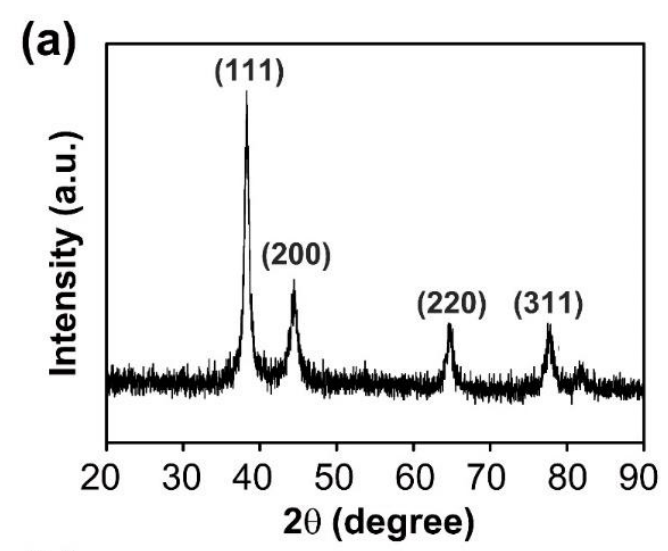

(b)

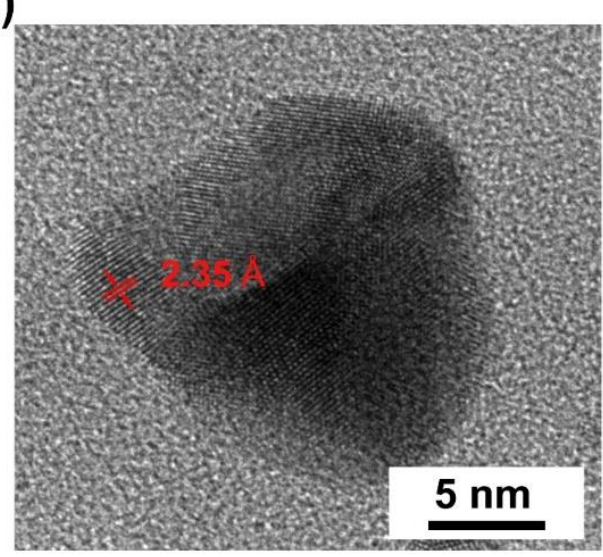

Figure S6. (a) The XRD pattern of the bowl-like spheres with Au NPs and (b) the HRTEM image of a Au NP in the bowl-like spheres. 

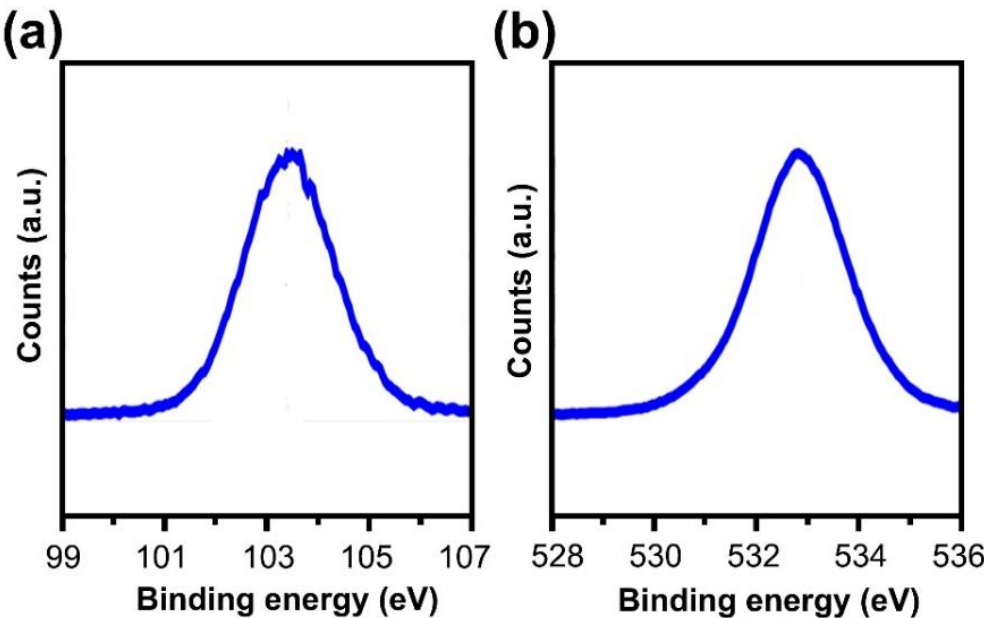

Figure S7. XPS (a) Si $2 p$ and (b) O $1 s$ of silica hollow spheres without Au NPs.
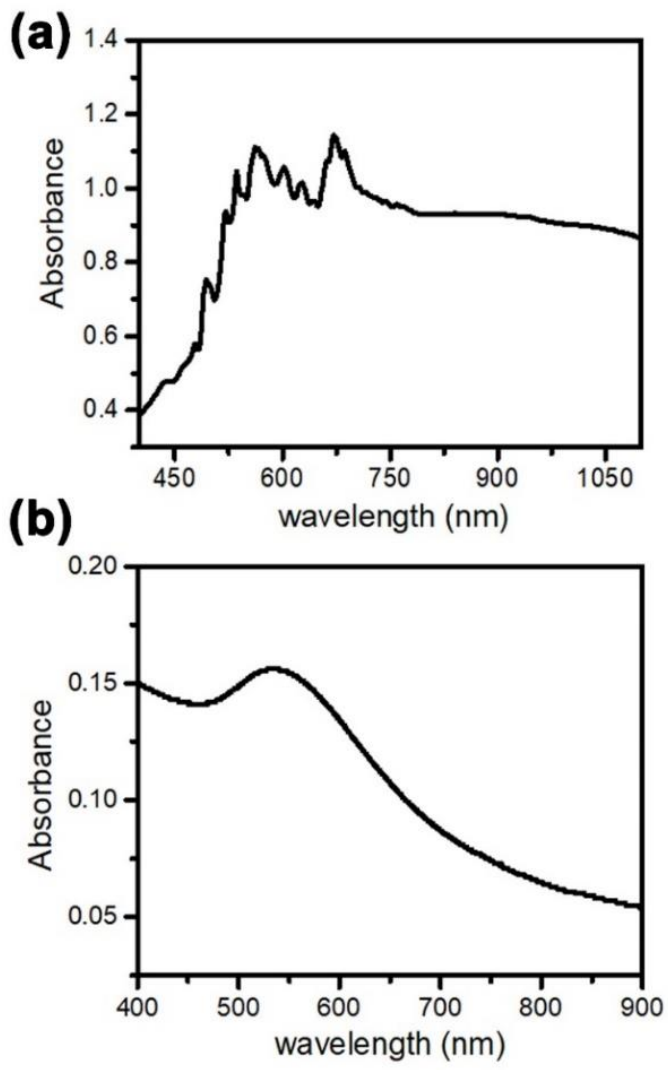

Figure S8. The UV-Vis spectra of (a) the bowl-like spheres with Au NPs (20-30 nm), and (b) the hollow silica spheres with post-loaded Au NPs (2-3 nm). 


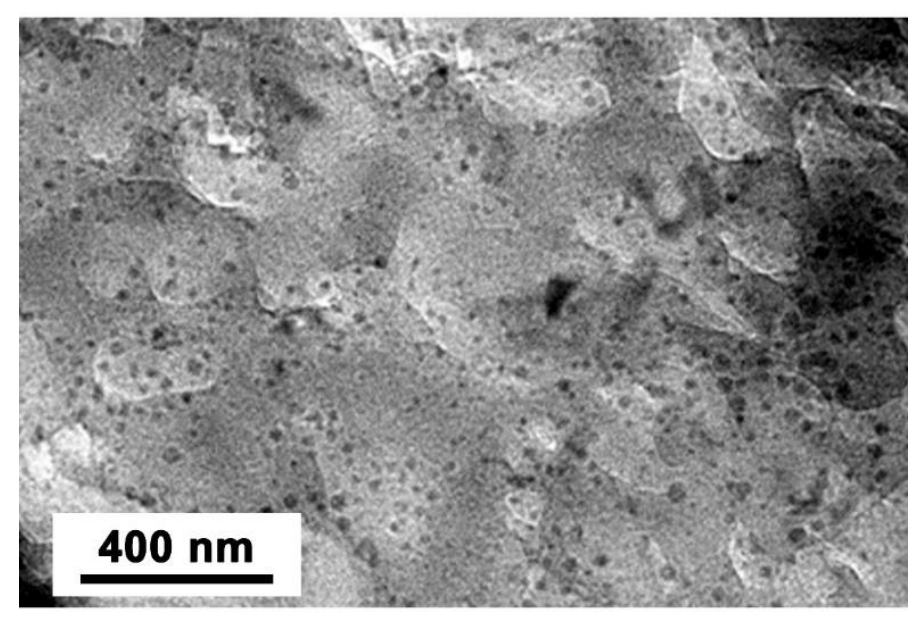

Figure S9. The TEM image of the post-loaded Au NPs $(2-3 \mathrm{~nm})$ on the surface of the silica nanosheets of the hollow silica spheres. 
(a)

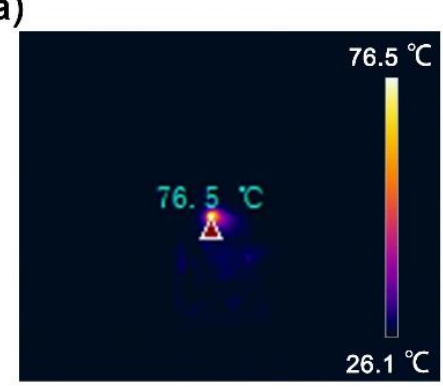

(b)

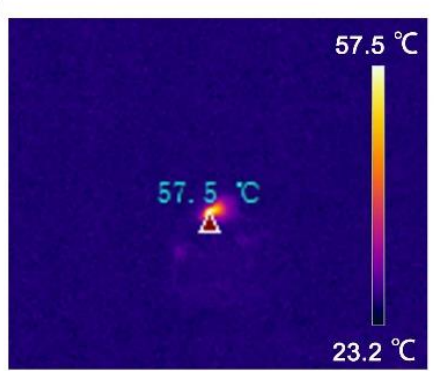

(c)

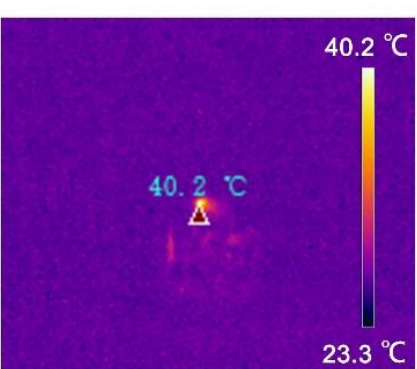

Figure S10. The thermal images of bowl-like spheres under the laser irradiation with

(a) $532 \mathrm{~nm}$, (b) $765 \mathrm{~nm}$ and (c) $1064 \mathrm{~nm}$ wavelength and at the power of $30 \mathrm{~mW}$.

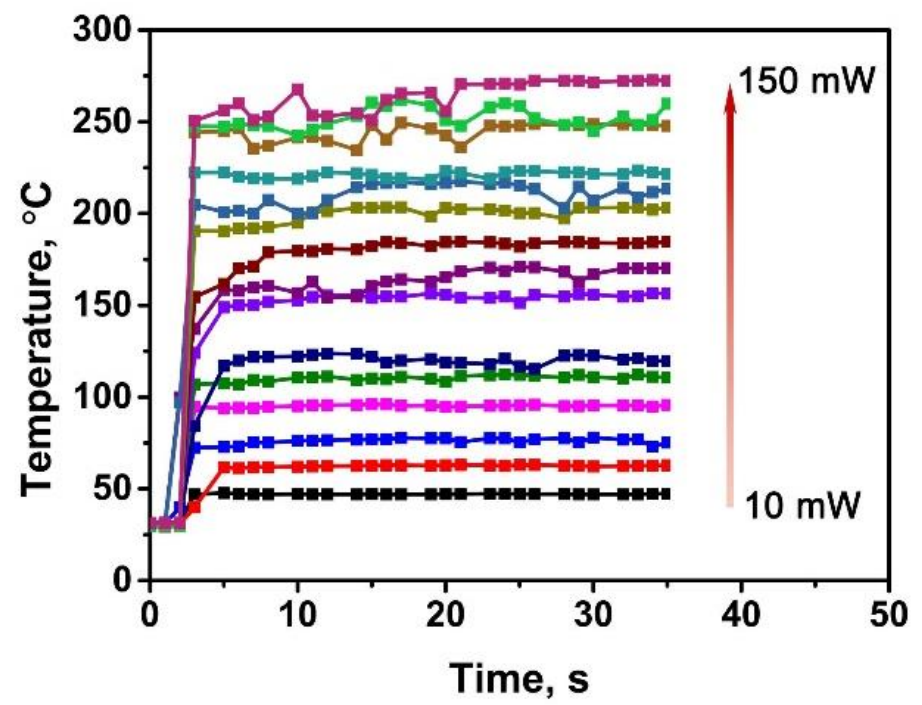

Figure S11. The real-time temperature changes of the bowl-like spheres under $532 \mathrm{~nm}$ laser irradiation at different powers. The test data were collected with every increase of $10 \mathrm{~mW}$ in the range of $10 \mathrm{~mW}-150 \mathrm{~mW}$. 


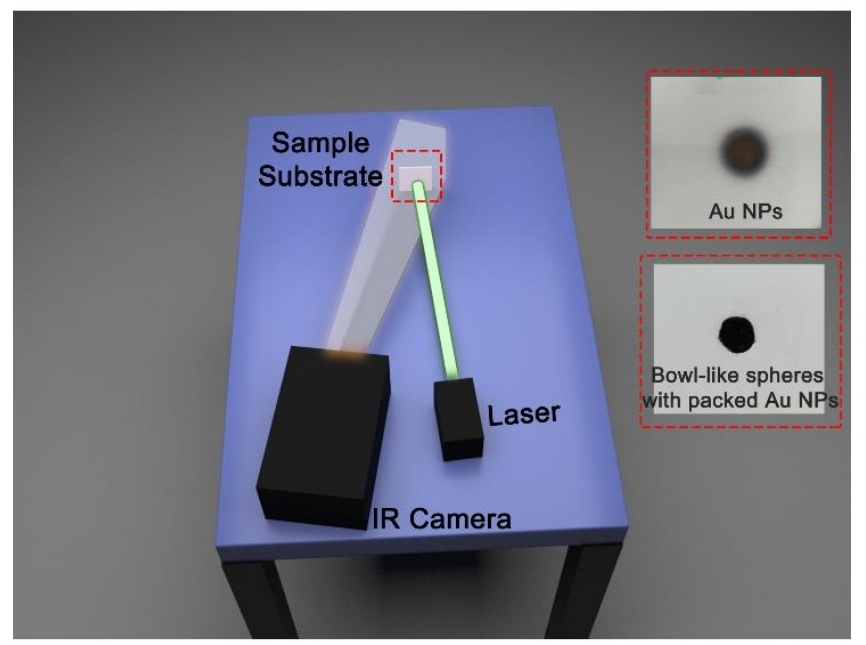

Figure S12. The scheme of the experimental setup for measuring photothermal conversion efficiency. The Au NPs were deposited on a thin layer chromatography plate by ion sputtering. The bowl-like spheres were dropped on a thin layer chromatography plate. 
(a)

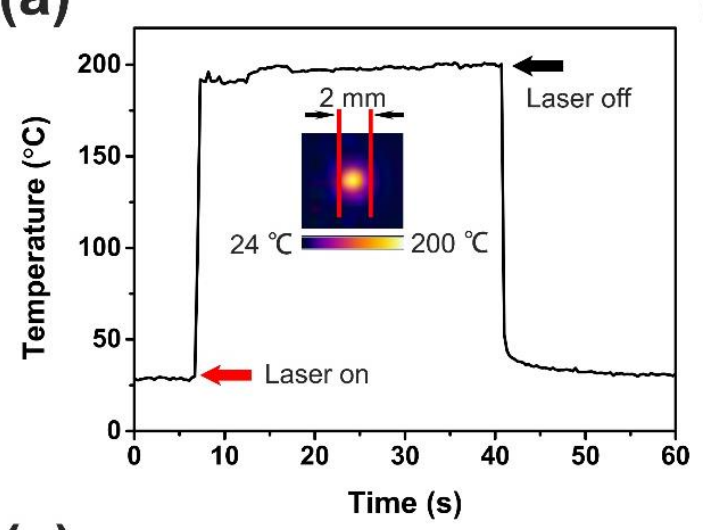

(c)

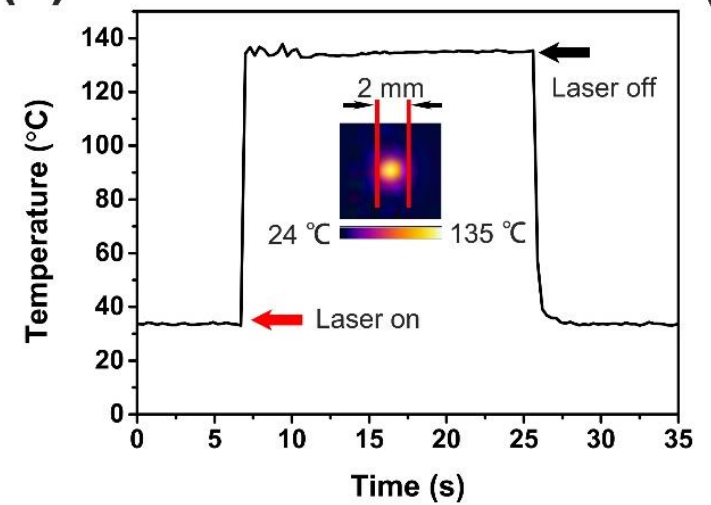

(b)

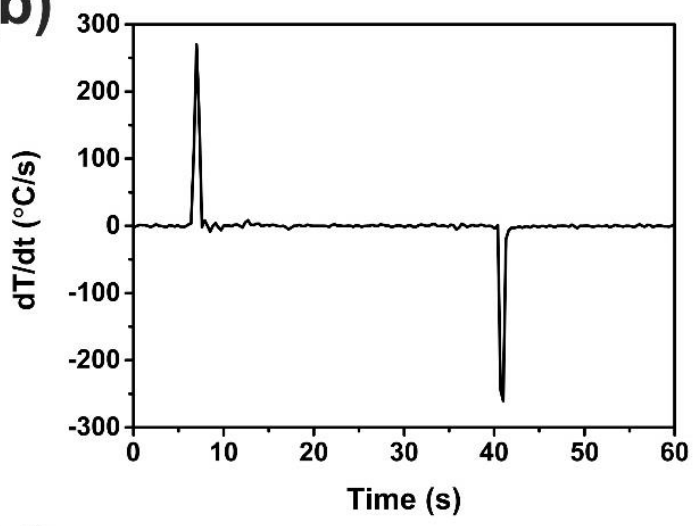

(d)

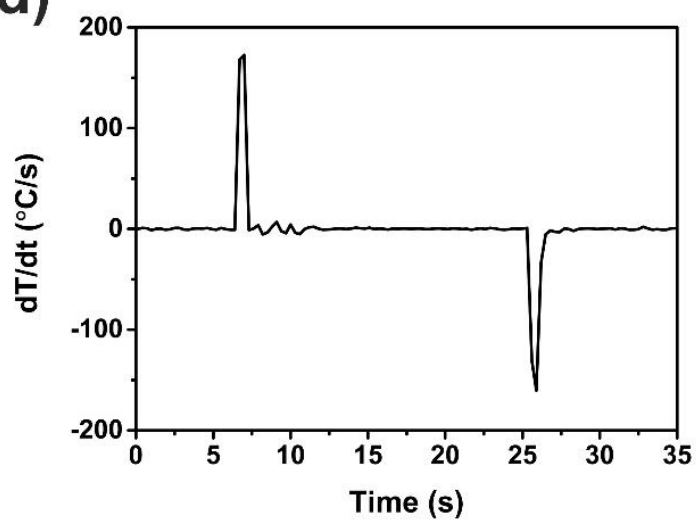

Figure S13. The temperature of (a) the bowl-like spheres and (b) the deposited Au NPs on the quartz plate upon the irradiation time. The temperature increasing rates of (c) the bowl-like spheres and (d) the deposited Au NPs on the quartz plate upon the irradiation time. The wavelength of laser is $532 \mathrm{~nm}$ and the light power is $90 \mathrm{~mW}$, respectively. The inset images showing the irradiation area.

The photothermal conversion process is analyzed by employing a theoretical model based on energy balance of the sample layers under laser irradiation. Photothermal conversion efficiency $(\eta)$ is used to quantitatively evaluate the capability of converting the laser into thermal energy and determined using equation $\mathrm{S} 1 \mathrm{R}^{\mathrm{R} 1}$ 


$$
\eta=\frac{Q}{E}=\frac{c m \Delta T}{P t}=\frac{\left(C_{\text {sample }} \times m_{\text {sample }}+C_{\text {silica }} \times m_{\text {silica }}\right)}{P} \times \frac{d T}{d t}
$$

where $Q$ denotes the photo-generated thermal energy and is calculated using heat capacity $(C)$ and mass $(m)$ of material as well as the temperature increment $(\Delta T)$ during laser irradiation. $E$ denotes the energy of incident laser and is determined from the power of laser $(P)$ and irradiation time $(t)$.

Typically, for the deposited Au NPs on the quartz plate, the mass of Au NPs ( $\mathrm{c}_{\mathrm{Au}}$, $\left.0.126 \mathrm{~J} \mathrm{~g}^{-1} \mathrm{~K}^{-1}\right)$ and silica gel $\left(C_{\text {silica }}, 0.921 \mathrm{~J} \mathrm{~g}^{-1} \mathrm{~K}^{-1}\right)$ within the irradiated zone can be estimated to be $122.46 \mu \mathrm{g}$ and $257.48 \mu \mathrm{g}$, respectively. The laser power is experimentally measured to be $90 \mathrm{~mW}$.

$$
\eta_{A u N P S}=\frac{(0.126 \times 122.46+0.921 \times 257.48) \times 10^{-6}}{90 \times 10^{-3}} \times 172 \times 100 \%=48.2 \%
$$

Similarly, the photothermal conversion efficiency of the bowl-like spheres can be calculated:

$$
\eta=\frac{(0.126 \times 112.5+0.921 \times 257.48) \times 10^{-6}}{90 \times 10^{-3}} \times 270 \times 100 \%=75.4 \%
$$

\section{Reference:}

S1. Z. Yang, X. Han, H. K. Lee, G. C. Phan-Quang, C. S. L. Koh, C. L. Lay, Y. H. Lee, Y. Miao, T. Liu, I. Y. Phang, X. Y. Ling. Shape-dependent thermo-plasmonic effect of nanoporous gold at the nanoscale for ultrasensitive heat-mediated remote actuation. Nanoscale, 2018, 10, 16005-16012. 
Table S1. Comparison of the photothermal performances of the bowl-like spheres and other reported gold-based plasmonic systems.

\begin{tabular}{|c|c|c|c|}
\hline $\begin{array}{l}\text { Au-Based Nanostructure } \\
\text { (Size) (Reporting Year) }\end{array}$ & $\begin{array}{l}\text { Irradiation } \\
\text { condition }\end{array}$ & $\begin{array}{c}\text { Temperature changes } \\
\Delta \Delta \mathrm{T}\left({ }^{\circ} \mathrm{C}\right)\end{array}$ & Reference \\
\hline Au nanourchins (90 nm) (2019) & $808 \mathrm{~nm}$ laser, $2 \mathrm{~W}$ & 40 (60 pM in water) & 17 \\
\hline $\begin{array}{c}\text { Au nanoparticles }(37.8 \mathrm{~nm}) \\
\text { (2017) }\end{array}$ & $\begin{array}{l}\text { Xenon lamp, } \\
150 \mathrm{~mW}\end{array}$ & 25 (in water) & 18 \\
\hline $\begin{array}{c}\text { Au bipyramid }(4.3 \mathrm{~nm}) \\
\text { supported in graphene oxide } \\
\text { (2013) }\end{array}$ & $532 \mathrm{~nm}$ laser, $4 \mathrm{~W}$ & 50 (in water) & 19 \\
\hline $\begin{array}{l}\text { Au nanorods (aspect ratio of } \\
\text { 3.8) (2012) }\end{array}$ & $808 \mathrm{~nm}$ laser, $1.8 \mathrm{~W}$ & 12 (in water) & 20 \\
\hline $\begin{array}{c}\text { Au nanocage (60 nm) } \\
@ \text { mSiO2@PNIPAM (2013) }\end{array}$ & $808 \mathrm{~nm}$ laser, $1 \mathrm{~W}$ & 18 (in water) & 21 \\
\hline $\begin{array}{c}\text { Au nanotriangles }(175 \mathrm{~nm}) \\
\text { (2017) }\end{array}$ & $785 \mathrm{~nm}$ laser, $2.5 \mathrm{~W}$ & 150 & 26 \\
\hline Au nanostars $(50 \mathrm{~nm})(2019)$ & $808 \mathrm{~nm}$ laser, $1 \mathrm{~W}$ & 23 (in water) & 12 \\
\hline $\begin{array}{c}\text { Au nanoporous bowl } \\
(700 \pm 100 \mathrm{~nm} \text { diameter })(2018)\end{array}$ & $532 \mathrm{~nm}, 90 \mathrm{~mW}$ & 113 & 14 \\
\hline $\begin{array}{c}\text { Au nanoporous tube }(200 \pm 100 \\
\text { nm diameter, } 6.1 \pm 2.3 \mu \mathrm{m} \\
\text { length) }(2018)\end{array}$ & $532 \mathrm{~nm}, 90 \mathrm{~mW}$ & 100 & 14 \\
\hline $\begin{array}{c}\text { Au nanoporous plate } \\
(2.0 \pm 0.5 \mu \mathrm{m} \text { edge length }) \\
(2018)\end{array}$ & $\begin{array}{l}532 \mathrm{~nm} \text { laser, } \\
90 \mathrm{~mW}\end{array}$ & 87 & 14 \\
\hline $\begin{array}{l}\text { The Bowl-like Spheres with } \mathrm{Au} \\
\text { NPs (20-30 nm diameter) }\end{array}$ & $\begin{array}{c}532 \mathrm{~nm} \text { laser, } \\
90 \mathrm{~mW} \\
532 \mathrm{~nm} \text { laser, } \\
150 \mathrm{~mW}\end{array}$ & 160 & This work \\
\hline
\end{tabular}


(a)

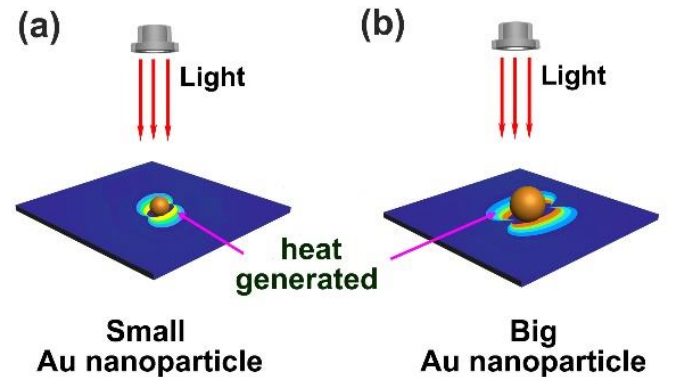

(c)

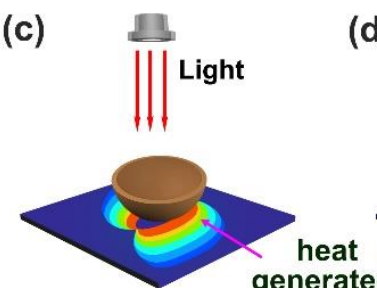

generated

Half-shelled

Au sphere (d)

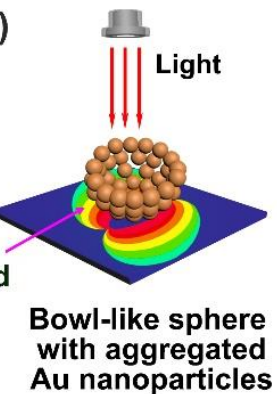

Scheme S1. The scheme for the comparison of light-induced heat effect by (a) small Au NPs, (b) big Au NPs, (c) half-shelled Au sphere, (d) bowl-like sphere with assembled Au NPs. 


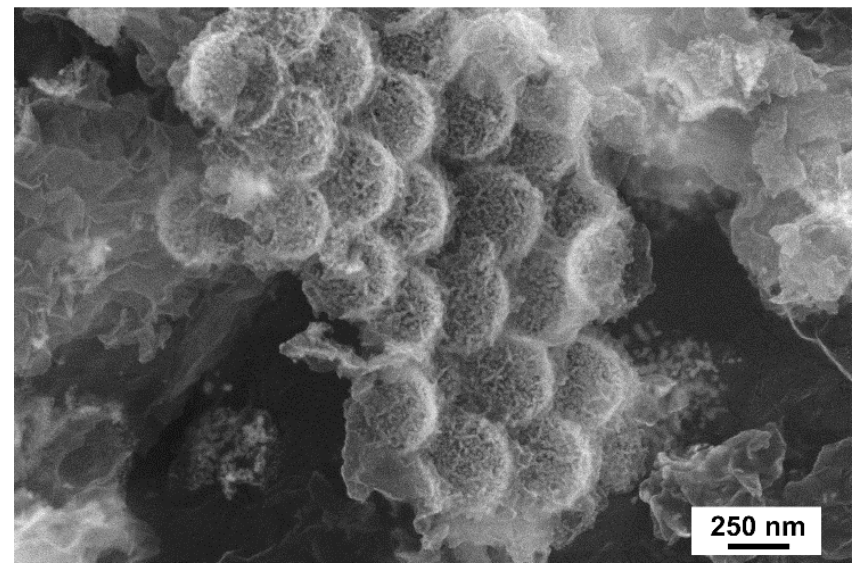

Figure S14. The SEM image of the bowl-like spheres after laser irradiation. 


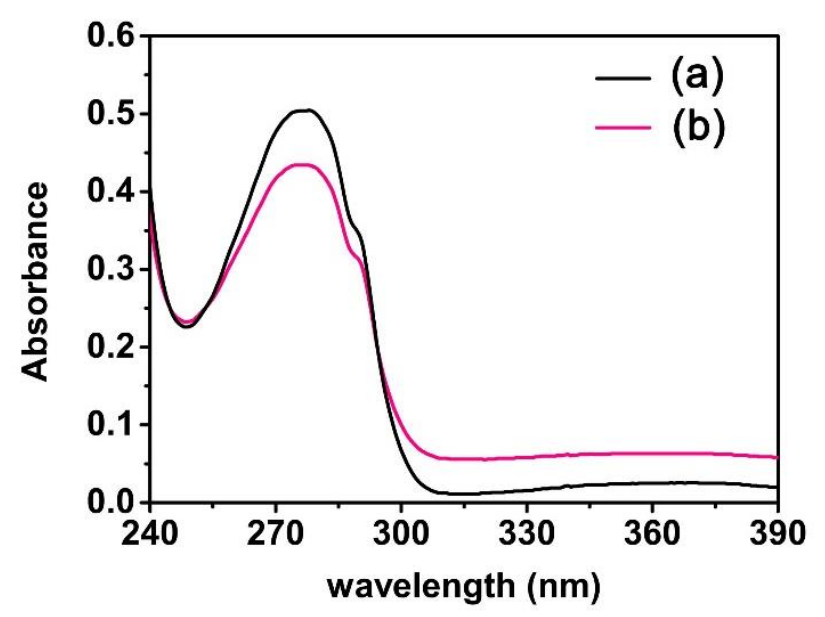

Figure S15. The UV-Vis spectra of (a) the COX solution and (b) the supernatant of the COX solution after mixing with the bowl-like spheres.

(a)

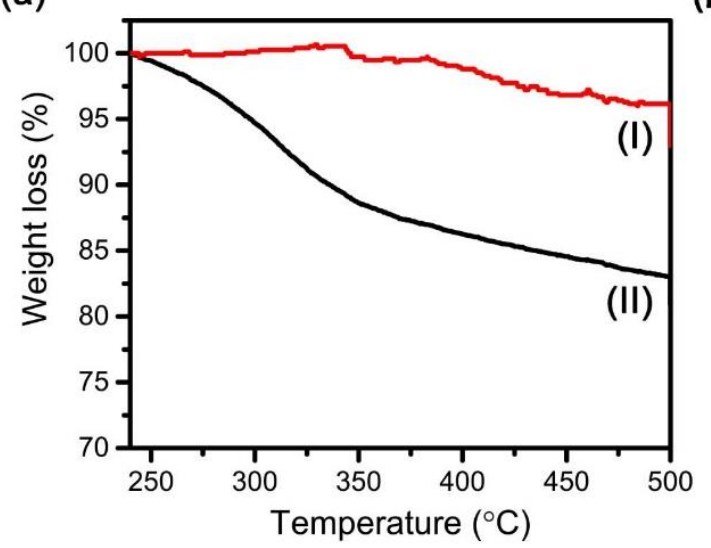

(b)

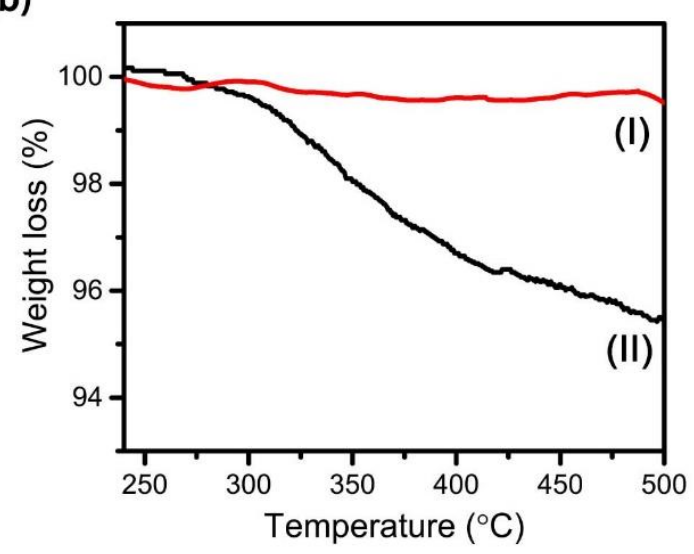

Figure S16. (a) The TGA weight loss of the silica hollow spheres (I) before and (II) after loaded with COX. (b) The TGA weight loss of the bowl-like spheres (I) before and (II) after loaded with COX. 
(a)
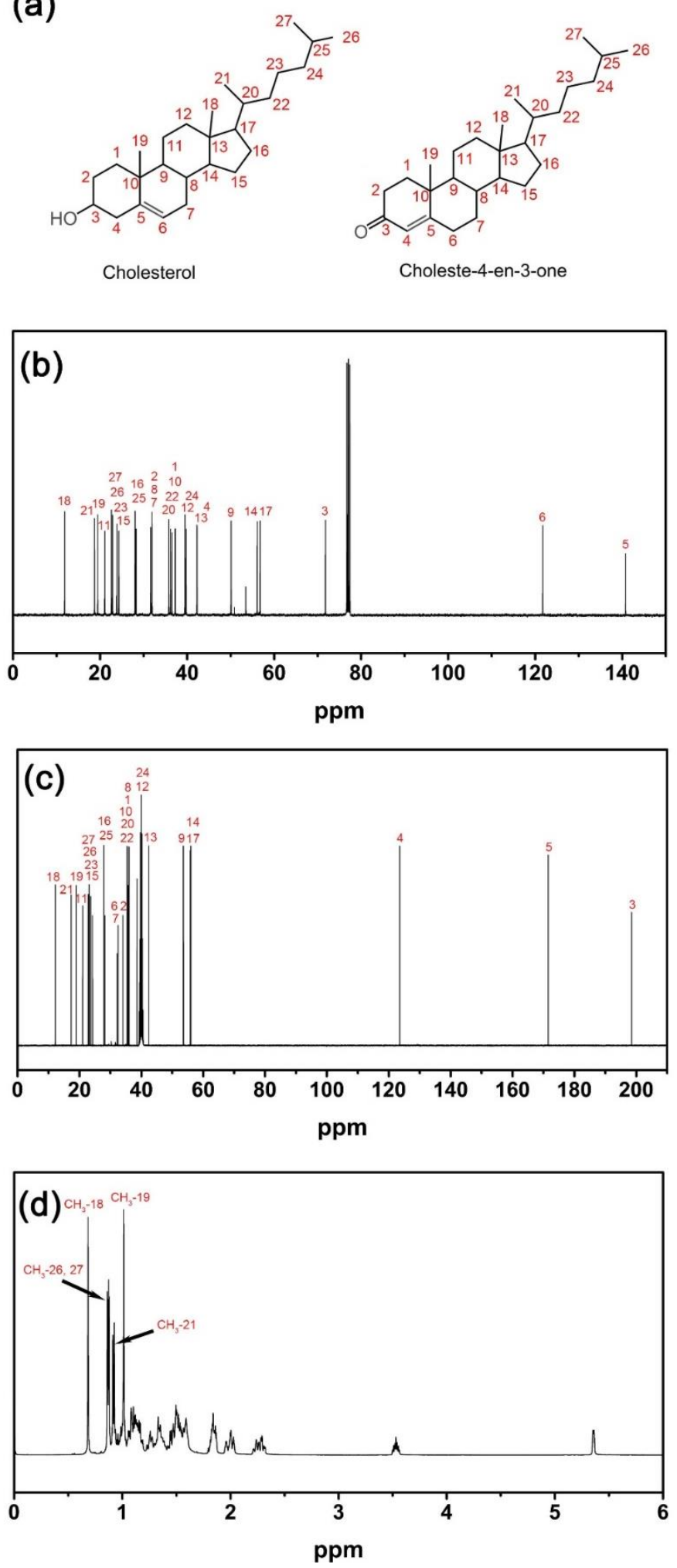

Figure S17. (a) The structures and nomenclatures of $\mathrm{C}$ for cholesterol and cholest-4en-3-one. (b) The ${ }^{13} \mathrm{C}$ NMR spectra of cholesterol. (c) The ${ }^{13} \mathrm{C}$ NMR spectra of cholest4-en-3-one. (d) The ${ }^{1} \mathrm{H}$ NMR spectra of cholest-4-en-3-one. 


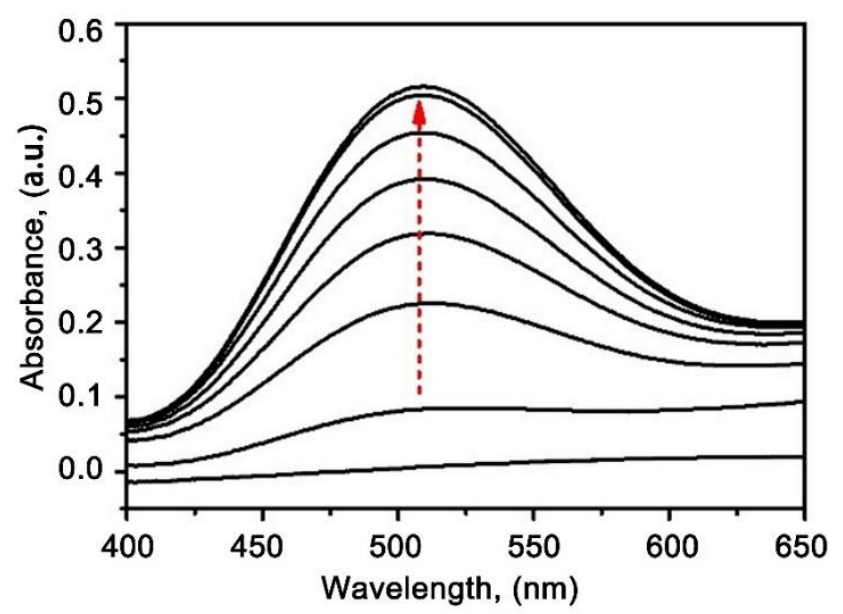

Figure S18. The UV-Vis spectra for the enzymatic reaction of COX in the bowl-like spheres at different reacting time. 


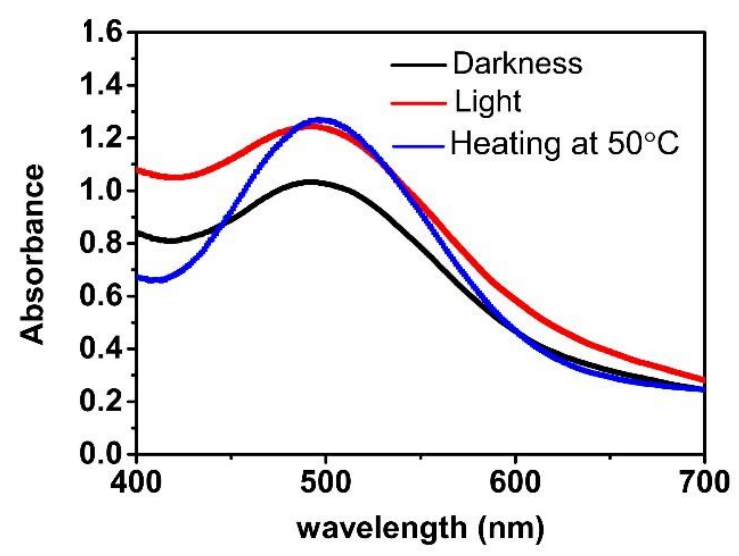

Figure S19. The UV-vis spectra of the cascade reaction solution catalyzed by COX in the bowl-like spheres with/without light irradiation, or heated to $50^{\circ} \mathrm{C}$ by hot plate.

According to the "Enzymatic activity assays", the concentration of cholesterol was $2.58 \mathrm{mM}$. The concentration of the product were $1.93 \mathrm{mM}$ and $1.43 \mathrm{mM}$ after the enzymatic reactions by COX in bowl-like spheres with and without light irradiation, respectively. Correspondingly, the cholesterol conversion rates were $75 \%$ and $55 \%$, respectively. 


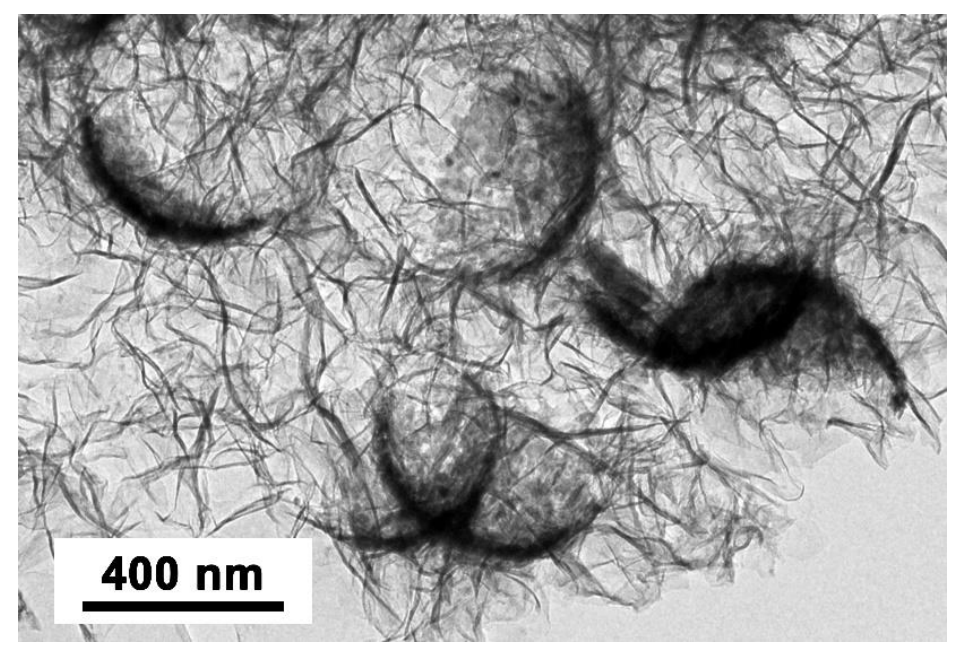

Figure S20. The TEM image of bowl-like spheres composed of packed $\mathrm{Cu}$ NPs, which fabricated by a similar process for bowl-like spheres composed by Au NPs. 


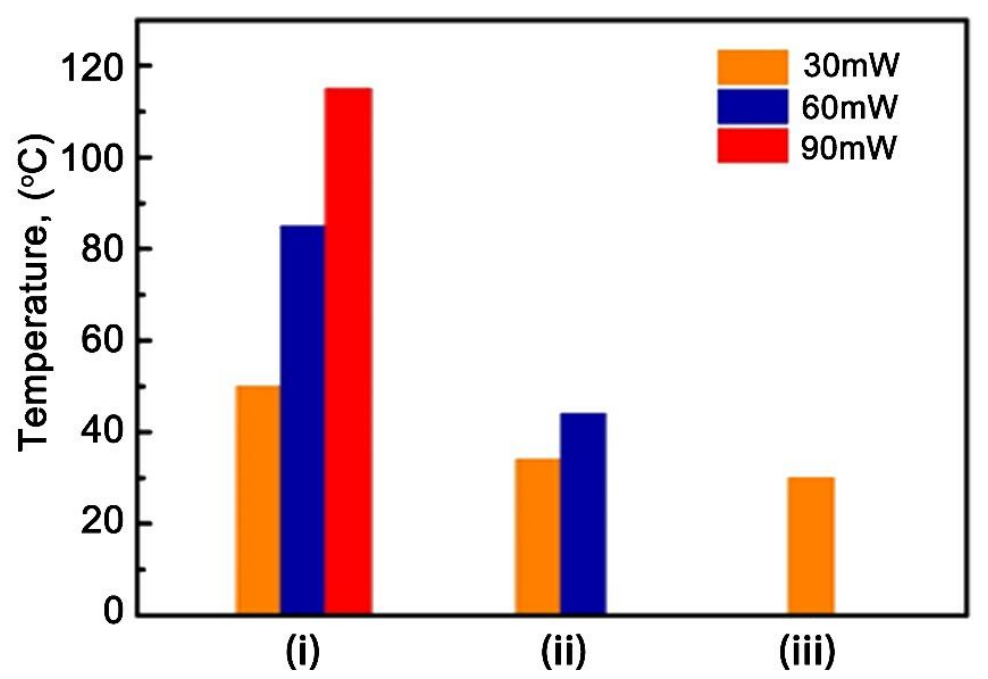

Figure S21. The temperature of the bowl-like spheres with $\mathrm{Cu}$ NPs upon laser irradiation with (i) $532 \mathrm{~nm}$, (ii) $785 \mathrm{~nm}$ and (iii) $1064 \mathrm{~nm}$ and the power of $30 \mathrm{~mW}, 60$ $\mathrm{mW}$ and $90 \mathrm{~mW}$. 